\title{
Efeito da veloplastia intravelar sobre o fechamento velofaríngeo avaliado por meio da técnica fluxo-pressão
}

\author{
Effect of intravelar veloplasty on velopharyngeal closure \\ assessed by pressure-flow technique
}

\author{
Renata Paciello Yamashita', Tathiane Roberta Teixeira Oliva², Ana Paula Fukushiro ${ }^{3}$, \\ Carolina Macedo Battaiola Brustello ${ }^{4}$, Inge Elly Kiemle Trindade ${ }^{5}$
}

\begin{abstract}
RESUMO
Objetivo: Avaliar o efeito da veloplastia intravelar sobre o fechamento velofaríngeo de pacientes com fissura de palato reparada e insuficiência velofaríngea (IVF), por meio de avaliação aerodinâmica (técnica fluxo-pressão). Métodos: Quarenta e sete indivíduos de ambos os gêneros, com idade entre seis e 48 anos, que apresentavam fissura de palato \pm lábio reparada e IVF, submetidos à veloplastia intravelar. Os pacientes foram avaliados, por meio da técnica fluxo-pressão, aferindo a medida da área do orifício velofaríngeo antes e, em média, 12 meses após a cirurgia, durante a produção do fonema /p/ inserido na palavra "rampa". O fechamento velofaríngeo foi classificado como adequado $\left(0\right.$ a $\left.0,049 \mathrm{~cm}^{2}\right)$, marginal $\left(0,050\right.$ a $\left.0,199 \mathrm{~cm}^{2}\right)$ ou inadequado $\left(\geq 0,200 \mathrm{~cm}^{2}\right)$. Resultados: Após a cirurgia, verificou-se melhora considerável no grau de fechamento velofaríngeo em $66 \%$ dos casos. Do total de pacientes analisados, $47 \%$ passaram a apresentar fechamento adequado, $23 \%$, fechamento marginal e $30 \%$ permaneceram com fechamento inadequado. Conclusão: Estes resultados mostraram que a veloplastia intravelar levou à melhora do fechamento velofaríngeo na maioria dos pacientes analisados repercutindo na redução dos sintomas de fala da IVF.
\end{abstract}

Descritores: Fissura palatina/cirurgia; Insuficiência velofaríngea/cirurgia; Rinomanometria/métodos; Fala; Palato mole

\section{INTRODUÇÃO}

A função velofaríngea é de fundamental importância na correta produção da fala. A ação esfinctérica das estruturas da velofaringe - palato mole e paredes faríngeas - é responsável

Trabalho desenvolvido no Laboratório de Fisiologia do Hospital de Reabilitação de Anomalias Craniofaciais da Universidade de São Paulo - USP - Bauru (SP), Brasil.

(1) Doutora, Fonoaudióloga do Laboratório de Fisiologia e do Setor de Fonoaudiologia do Hospital de Reabilitação de Anomalias Craniofaciais da Universidade de São Paulo - HRAC-USP - Bauru (SP), Brasil.

(2) Pós-graduanda (Especialização) em Motricidade Orofacial do Hospital de Reabilitação de Anomalias Craniofaciais da Universidade de São Paulo USP - Bauru (SP), Brasil.

(3) Doutora, Professora do Departamento de Fonoaudiologia da Faculdade de Odontologia de Bauru da Universidade de São Paulo - USP - Bauru (SP), Brasil.

(4) Pós-Graduanda (Doutorado) em Ciências da Reabilitação do Programa de Pós-Graduação do Hospital de Reabilitação de Anomalias Craniofaciais da Universidade de São Paulo - USP - Bauru (SP), Brasil.

(5) Professora Titular do Departamento de Ciências Biológicas da Faculdade de Odontologia de Bauru da Universidade de São Paulo e do Hospital de Reabilitação de Anomalias Craniofaciais (HRAC) da Universidade de São Paulo - USP - Bauru (SP), Brasil.

Endereço para correspondência: Renata Paciello Yamashita. R. Sílvio Marchione, 3-20, Vila Universitária, Bauru (SP), Brasil, CEP: 17012-900.

E-mail: rezeyama@usp.br

Recebido em: 22/9/2009; Aceito em: 30/10/2009 pela distribuição do fluxo aéreo expiratório e das vibrações acústicas para a cavidade oral, na produção dos sons orais e para a cavidade nasal, na produção dos sons nasais. Quando estas estruturas falham em realizar o fechamento velofaríngeo em função de alterações de origem estrutural, tem-se a insuficiência velofaríngea (IVF). Nestas condições, parte da corrente aérea sonorizada é desviada para a cavidade nasal, comprometendo de diferentes formas a produção da fala. Os sintomas mais comuns da IVF são: hipernasalidade, que é caracterizada pelo excesso de ressonância nasal na produção de sons normalmente não nasalizados, como resultado da incapacidade do esfíncter velofaríngeo manter-se fechado o suficiente para evitar a ressonância nasal dos sons articulados oralmente; a emissão de ar nasal, caracterizada pelo inapropriado escape de ar pelo nariz que ocorre durante a produção de consoantes de pressão, podendo ser audível ou não; e os distúrbios articulatórios compensatórios, que são considerados estratégias, desenvolvidas pelo indivíduo, substituindo os sons articulados oralmente por sons articulados em locais posteriores ou superiores ao esfíncter velofaríngeo, a fim de compensar a incapacidade de impor pressão na cavidade oral e evitar a perda de pressão durante a fala ${ }^{(1,2)}$.

A causa mais freqüente da IVF é a fissura palatina na qual a inserção dos músculos do palato e, em particular, do músculo levantador do véu palatino, está direcionada anteriormente, 
permanecendo em uma posição sagital, inserido na borda posterior do palato duro. Desse modo, não há formação da cinta muscular necessária ao fechamento velofaríngeo, levando à alteração das forças vetoriais resultantes ${ }^{(1,3,4)}$.

Apesar do reconhecido esforço para normalizar a orientação muscular na correção primária do palato, certa parcela de pacientes submetidos à palatoplastia primária pode permanecer com sintomas da IVF, sendo uma das possíveis causas a desorientação das fibras musculares e da sua inserção anteriorizada na borda posterior das lâminas palatinas ${ }^{(1,5-7)}$. Nestes casos torna-se necessária uma cirurgia secundária do palato. Dentre as técnicas cirúrgicas empregadas para esse fim, várias utilizam o procedimento conhecido como veloplastia intravelar, o qual tem como objetivo reposicionar a musculatura do palato mole oferecendo boa mobilidade ao véu palatino e conseqüente melhora da competência velofaríngea. Tal procedimento cirúrgico vem sendo amplamente divulgado na literatura, como uma opção para a correção da IVF residual ${ }^{(3,5,6,8-10)}$.

O ponto mais importante da veloplastia intravelar é a total liberação da musculatura do palato que está inserida ao longo da borda posterior do osso palatino em ambos os lados da espinha nasal posterior, conforme ilustrado na Figura 1A. Esta liberação é fundamental para o resultado funcional da cirurgia. $\mathrm{O}$ feixe muscular é então dirigido medial e posteriormente para permitir uma posição mais transversal de suas fibras (Figura 1B). Com este procedimento realizado bilateralmente, há a possibilidade de uma sobreposição discreta dos feixes musculares em uma posição bem mais posterior, mais próxima à condição fisiológica normal ${ }^{(3,11)}$.

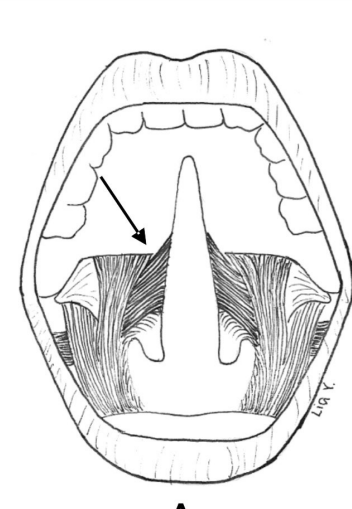

A

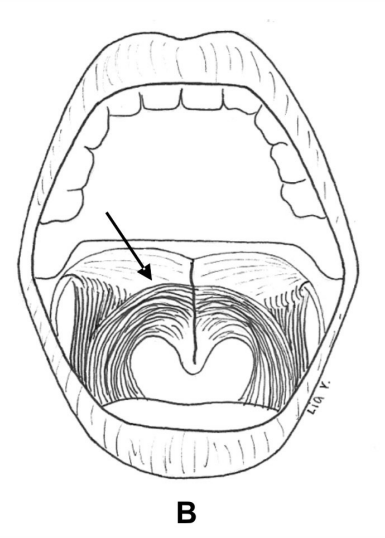

Figura 1. Representação esquemática da musculatura do palato mole. Obs.: A seta $A$ indica a inserção da musculatura na borda posterior do osso palatino (posição sagital); a seta B indica a musculatura reposicionada e posteriorizada (posição transversal).

Vários estudos têm demonstrado a eficiência da veloplastia intravelar na correção da $\operatorname{IVF}^{(5,6,8-10,12-15)}$. A maioria deles utilizou avaliação perceptiva da fala para classificar a hipernasalidade; a emissão de ar nasal, a inteligibilidade de fala e/ ou métodos instrumentais diretos tais como a nasoendoscopia e a videofluoroscopia, para avaliar os resultados da cirurgia e suas repercussões sobre a fala ${ }^{(6,10)}$.

Em estudo preliminar realizado no Laboratório de Fisiologia do HRAC-USP, utilizando avaliação perceptiva e nasométrica $^{(16)}$, foi verificado o efeito, a curto prazo (em média oito meses), da veloplastia intravelar associada à palatoplastia secundária sobre a fala de pacientes com IVF. Foram organizados dois grupos, de acordo com a condição nasoendoscópica pré-cirúrgica: pacientes com falhas pequenas no fechamento velofaríngeo (gaps pequenos) e pacientes com falhas de tamanho médio e grande no fechamento velofaríngeo (gaps médios e grandes). Concluiu-se que a cirurgia foi mais eficiente na melhora e resolução dos sintomas de fala nos pacientes com falhas pequenas do fechamento velofaríngeo. Entretanto, verificou-se que a cirurgia levou à melhora, embora em menor proporção, da fala daqueles pacientes que apresentavam falhas grandes no fechamento velofaríngeo. Segundo a literatura, mesmo os casos que permanecem com IVF após a cirurgia secundária se beneficiam da veloplastia intravelar, pois o reposicionamento da musculatura do palato favorece o movimento velar, o que pode torná-los candidatos a outras intervenções cirúrgicas menos agressivas no futuro ${ }^{(6,10)}$.

Como recomendação da American Cleft Palate Association $^{(17)}$, a aferição dos resultados das cirurgias para correção da IVF deve envolver pelo menos um dos métodos de avaliação instrumental do mecanismo velofaríngeo, a saber, a nasoendoscopia, a videofluoroscopia, a nasometria ou a técnica fluxo-pressão. Esta última, em particular, permite determinar a área do orifício velofaríngeo conhecendo-se a diferença de pressão existente entre os lados do orifício e do fluxo aéreo que o atravessa e assim, estimar a função velofaríngea de maneira não invasiva. Até hoje, tal método não foi empregado na avaliação dos resultados cirúrgicos da veloplastia intravelar. Por outro lado, a literatura já demonstrou a eficácia da técnica fluxo-pressão na avaliação e acompanhamento dos resultados cirúrgicos do retalho faríngeo e da esfincteroplastia ${ }^{(18-24)}$.

Uma vez que a veloplastia intravelar secundária é realizada para proporcionar boa mobilidade do palato e estabelecer condições anatômicas para o fechamento velofaríngeo adequado, o presente estudo teve como finalidade avaliar objetivamente, por meio de avaliação aerodinâmica, o efeito desta cirurgia sobre o fechamento velofaríngeo de pacientes com fissura de palato reparada e IVF residual.

\section{MÉTODOS}

O presente estudo foi desenvolvido no Laboratório de Fisiologia do Hospital de Reabilitação de Anomalias Craniofaciais (HRAC-USP), após aprovação do Comitê de Ética em Pesquisa com Seres Humanos da instituição (ofício ${ }^{\circ}$ 045/2008-SVAPEPE-CEP). Todos os pacientes incluídos no estudo foram selecionados entre aqueles que estavam aguardando internação para a palatoplastia secundária associada à veloplastia intravelar para correção da IVF. Foram avaliados 47 indivíduos, de ambos os sexos, com idades entre seis e 48 anos (média=16 anos), com fissura de palato associada ou não à fissura de lábio, já operada. Não foram incluídos no estudo pacientes com síndromes e/ou problemas neurológicos evidentes, com sintomas respiratórios alérgicos agudos que resultassem em congestão nasal ao exame.

Todos os pacientes foram submetidos à avaliação aerodinâ- 
mica por meio da técnica fluxo-pressão, para medida da área do orifício velofaríngeo, dois dias antes, em média, da cirurgia (pré) e 12 meses, em média, após a cirurgia (pós).

Foi utilizado o sistema computadorizado PERCI-SARS (versão 3.30, Microtronics Corp., Chapel Hill, NC USA). A técnica fluxo-pressão baseia-se no fato de que a área de secção transversa de uma constrição (ou orifício) pode ser estimada pela medida simultânea da pressão diferencial entre os dois lados da constrição, e, do fluxo aéreo que a atravessa ${ }^{(25)}$.

Sendo assim, a área do orifício velofaríngeo foi determinada durante a produção do fonema plosivo /p/, inserido no vocábulo "rampa", posicionando-se um cateter no interior da cavidade oral e outro na narina de menor fluxo nasal. Esse cateter é mantido em posição por um obturador nasal. Ambos os cateteres medem pressões aéreas estáticas que são transmitidas a transdutores de pressão. O fluxo aéreo nasal é medido, por meio de um tubo plástico, adaptado à narina de maior fluxo, conectado a um pneumotacógrafo previamente aquecido e também ligado a um transdutor de pressão. Os sinais dos três transdutores (pressão nasal, pressão oral e fluxo nasal) são enviados ao sistema para análise por software específico. A calibração do sistema é feita antes de cada exame com sinais conhecidos de fluxo $(250 \mathrm{ml} / \mathrm{s})$ e pressão $\left(6 \mathrm{cmH}_{2} \mathrm{O}\right)$, utilizando, respectivamente, um rotâmetro e um manômetro de água (Figura 2).

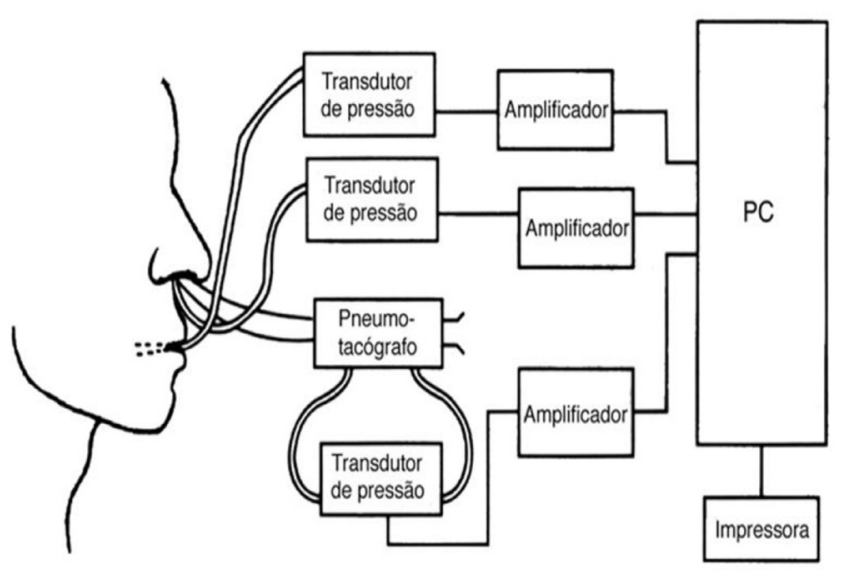

Fonte: Trindade IE, Silva Filho MG. Fissuras labiopalatinas: uma abordagem interdisciplinar. São Paulo: Editora Santos; 2007. Diagnóstico instrumental da disfunção velofaríngea. p. 137.

Figura 2. Instrumentação para determinação da área do orifício velofaríngeo (Sistema PERCI-SARS, Microtronics Corp., Chapel Hill, NC, USA)

Foram avaliadas de quatro a seis emissões sucessivas de cada produção por indivíduo. A área considerada para análise representa a média dessas produções múltiplas e foi calculada pelo próprio programa a partir da equação: $\mathrm{A}=\dot{\mathrm{V}} / \mathrm{k}$ $(2 \Delta \mathrm{P} / \mathrm{d})^{1 / 2}$, onde $\mathrm{A}=$ área do orifício em $\mathrm{cm}^{2} ; \dot{\mathrm{V}}=$ fluxo nasal $\mathrm{em} \mathrm{ml} / \mathrm{s} ; \mathrm{k}=0,65 ; \Delta \mathrm{P}=$ pressão oral - pressão nasal em $\mathrm{cmH}_{2} \mathrm{O}$; $\mathrm{d}=$ densidade do ar em $\mathrm{g} / \mathrm{cm}^{3}$. Nenhum paciente apresentava articulação compensatória na produção do fonema /p/, confirmada pela avaliação perceptiva da fala pré-operatória.

O fechamento velofaríngeo foi classificado de acordo com critério adaptado de Warren ${ }^{(26)}$ baseado nos valores da área ve- lofaríngea, onde: 0 a $0,049 \mathrm{~cm}^{2}=$ fechamento velofaríngeo adequado; 0,050 a $0,199 \mathrm{~cm}^{2}=$ fechamento velofaríngeo marginal e, $0,200 \mathrm{~cm}^{2}$ ou mais=fechamento velofaríngeo inadequado.

Para a área do orifício velofaríngeo foi calculada a mediana e a significância da diferença pré e pós-operatória foi investigada por meio do teste não paramétrico de Wilcoxon, ao nível de significância de $1 \%$. O sucesso cirúrgico foi, também, avaliado em termos de melhora e normalização do fechamento velofaríngeo.

\section{RESULTADOS}

Antes da cirurgia, a mediana obtida para a área do orifício velofaríngeo foi de $0,792 \mathrm{~cm}^{2}\left(1^{\circ}\right.$ quartil $=0,371 \mathrm{~cm}^{2} \mathrm{e}$ $3^{\circ}$ quartil $=0,800 \mathrm{~cm}^{2}$ ), indicativo de fechamento velofaríngeo inadequado. Após a cirurgia, houve redução do valor da área mediana para $0,053 \mathrm{~cm}^{2}\left(1^{\circ}\right.$ quartil $=0,011 \mathrm{~cm}^{2} \mathrm{e}$ $3^{\circ}$ quartil $=0,414 \mathrm{~cm}^{2}$ ), indicando fechamento velofaríngeo marginal, porém muito próximo ao valor de normalidade, conforme critério adotado no estudo. A análise dos dados mostrou que houve diferença entre os valores pré e pósoperatórios (Tabela 1).

Tabela 1. Valores da área dos orifícios velofaríngeos obtidos antes e após a cirurgia de veloplastia intravelar e suas diferenças

\begin{tabular}{llccc}
\hline Área & $\mathrm{N}$ & Mediana & $1^{\circ}$ quartil & $3^{\circ}$ quartil \\
\hline Pré & 47 & 0,792 & 0,371 & 0,800 \\
Pós & 47 & $0,053^{*}$ & 0,011 & 0,414 \\
\hline
\end{tabular}

Teste de Wilcoxon. ${ }^{*}$ Valores significativos $(p<0,01)$

Legenda: $\mathrm{N}=$ número de sujeitos

A proporção dos pacientes com valores de área dos orifícios velofaríngeos indicativos de função velofaríngea marginal, antes da cirurgia, foi de $13 \%$ (6) e inadequada de $87 \%$ (41). Após a cirurgia, $47 \%$ (22) dos pacientes passaram a apresentar função velofaríngea adequada, $23 \%$ (11) função velofaríngea marginal e $30 \%$ (14) permaneceram com função velofaríngea inadequada.

Tabela 2. Distribuição dos pacientes de acordo com a classificação do fechamento velofaríngeo na avaliação aerodinâmica durante a produção do fonema /p/ inserido no vocábulo "rampa", antes (pré) e após (pós) a cirurgia

\begin{tabular}{lcccc}
\hline Pós & \multicolumn{3}{c}{ Pré } \\
\cline { 2 - 4 } & $\mathrm{A}(\%)$ & $\mathrm{M}(\%)$ & $\mathrm{I}(\%)$ & Total \\
\hline $\mathrm{A}$ & 0 & $4(9)$ & $18(38)$ & $22(47)$ \\
$\mathrm{M}$ & 0 & $2(4)$ & $9(19)$ & $11(23)$ \\
$\mathrm{I}$ & 0 & 0 & $14(30)$ & $14(30)$ \\
\hline Total & 0 & $6(13)$ & $41(87)$ & $47(100)$ \\
\hline
\end{tabular}

Legenda: $A$ = adequado; $M=$ marginal; $I$ = inadequado

Obs.: Em destaque encontram-se os pacientes com melhora do fechamento velofaríngeo.

Analisando o sucesso cirúrgico, verificou-se melhora no fechamento velofaríngeo caracterizada pela diminuição da área do orifício velofaríngeo durante a fala, em $66 \%$ (31/47) dos pacientes. Em 34\% (16/47) dos pacientes o fechamento velofaríngeo manteve-se inalterado (Figura 3). 


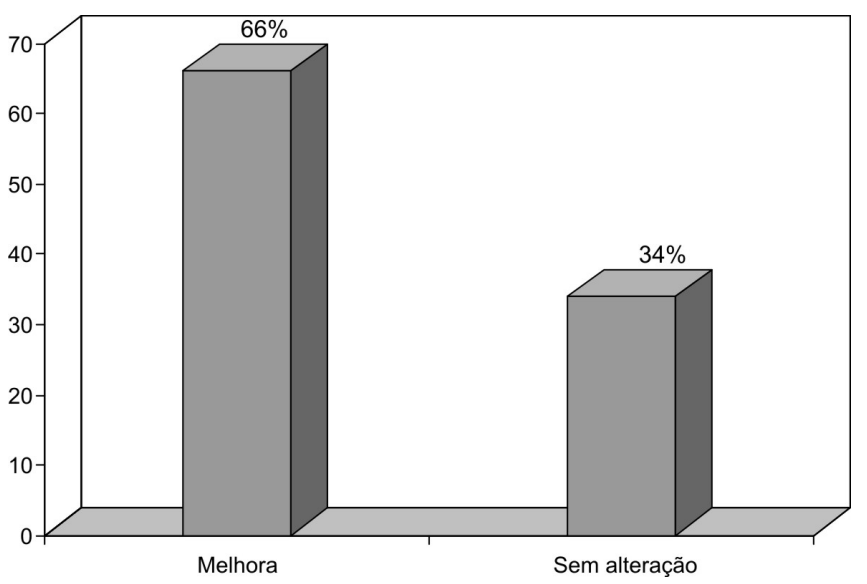

Figura 3. Distribuição dos pacientes de acordo com o efeito da cirurgia sobre o fechamento velofaríngeo, avaliado pela técnica fluxo-pressão

\section{DISCUSSÃO}

Nos últimos anos, a veloplastia intravelar tem sido utilizada para o tratamento cirúrgico da IVF no HRAC-USP, a exemplo de centros craniofaciais do mundo, por proporcionar mobilidade ao palato mole, preservar a anatomia velofaríngea e, assim, não apresentar riscos de comprometimento das vias aéreas superiores quando comparada a outras técnicas cirúrgicas, como o retalho faríngeo ou a esfincteroplastia. A escolha pela veloplastia intravelar, na palatoplastia secundária para correção da IVF residual, está na dependência da anatomia e fisiologia da velofaringe identificadas na avaliação instrumental pré-cirúrgica e da experiência prévia do cirurgião. $\mathrm{O}$ principal critério a ser considerado na seleção de pacientes com IVF residual deve ser a inserção anteriorizada da musculatura do palato e gap velofaríngeo de pequena extensão, que, para alguns autores, não deve ultrapassar $5 \mathrm{~mm}^{(5)}$.

Um dos grandes desafios dos clínicos e pesquisadores em fissura é estabelecer fatores indicativos para a realização das diversas técnicas cirúrgicas existentes na correção da IVF. A correta seleção das características a serem consideradas na indicação da cirurgia é essencial para o seu sucesso. A gravidade da IVF é um importante fator na escolha do procedimento cirúrgico a ser empregado. O que está estabelecido atualmente é que a veloplastia intravelar é indicada nos casos de IVF de menor gravidade, em pacientes com gaps velofaríngeos pequenos e/ou função velofaríngea marginal, enquanto que o retalho faríngeo é indicado nos casos de IVF grave, ou seja, naqueles pacientes com gaps grandes no fechamento velofaríngeo. Entretanto, a literatura tem mostrado que a veloplastia intravelar é também efetiva, na presença de IVF grave ${ }^{(6,8)}$. Uma vez que o objetivo da cirurgia é modificar a inserção da musculatura velar para melhorar a função velofaríngea, seria esperado que o uso deste procedimento nos pacientes que apresentam gaps médios e grandes, também pudesse levar a alguma melhora da fala, ainda que não atingindo a normalidade. No presente estudo, 47 pacientes com gaps de extensões variadas foram elegíveis à veloplastia intravelar, com base na avaliação perceptiva e nasofaringoscópica prévias, e foram avaliados utilizando-se a técnica fluxo-pressão antes e após a cirurgia.
De modo geral, a veloplastia intravelar levou a mudanças positivas no fechamento velofaríngeo em $66 \%$ dos pacientes do presente estudo. Após a cirurgia, a mediana obtida para a área do orifício velofaríngeo foi de $0,053 \mathrm{~cm}^{2}$, que, segundo a classificação adotada corresponde ao fechamento velofaríngeo marginal. Porém, este valor está muito próximo do limite correspondente ao fechamento adequado $\left(0,049 \mathrm{~cm}^{2}\right)$. A análise individual dos dados mostrou que $47 \%$ dos pacientes passaram a apresentar fechamento velofaríngeo adequado, determinado pela técnica fluxo-pressão, sugerindo eliminação da hipernasalidade.

Proporção superior de $89 \%$ de pacientes com fechamento velofaríngeo completo após a veloplastia intravelar foi encontrada em um estudo que utilizou metodologia perceptiva, nasoendoscópica e videofluoroscópica ${ }^{(5)}$. Entretanto, há que se considerar que esse elevado índice de sucesso pode ser explicado pelo critério de indicação cirúrgica de gap velofaríngeo menor que $5 \mathrm{~mm}$ adotado por esses autores. Proporções igualmente superiores foram encontradas em outros dois estudos utilizando a mesma metodologia. O primeiro, relatou significante melhora do fechamento velofaríngeo em $88 \%$ dos pacientes após a veloplastia intravelar ${ }^{(9)}$ e o segundo, utilizando avaliação perceptiva e instrumental, encontrou expressiva melhora da função velofaríngea em $87 \%$ e função velofaríngea dentro dos limites normais em $56 \%$ dos pacientes estudados ${ }^{(10)}$. Já outros estudos que avaliaram os efeitos da cirurgia por meio de julgamento perceptivo e nasofaringoscópico, verificaram redução do tamanho do gap velofaríngeo com eliminação da IVF em proporção semelhante, de $40 \%$, dos pacientes ${ }^{(6)}$.

$\mathrm{O}$ efeito da veloplastia intravelar sobre a fala de pacientes com IVF foi demonstrado em estudo realizado no Laboratório de Fisiologia em 40 pacientes $^{(16)}$. A autora utilizou avaliação perceptiva da fala e nasometria e verificou que a veloplastia intravelar levou à melhora da fala em parcela considerável de pacientes, ainda que a curto prazo. $\mathrm{O}$ estudo mostrou que a cirurgia foi mais eficiente na redução da hipernasalidade em pacientes com gaps velofaríngeos pequenos (84\% dos casos). Entretanto, verificou que em pacientes com gaps grandes também houve melhora, embora em menor proporção (73\%), mostrando que a veloplastia intravelar pode beneficiar, também, aqueles pacientes que apresentam IVF mais grave. Ainda que no presente estudo, os pacientes tenham sido avaliados como um único grupo incluindo todos os tamanhos de gap, o índice de sucesso foi semelhante.

Resultados variáveis com proporções de melhora dos sintomas de fala de 52 a $93 \%$ foram obtidos em estudos que investigaram outras técnicas cirúrgicas para a correção da IVF, como o retalho faríngeo e a esfincteroplastia, utilizando diferentes metodologias. Proporções inferiores a do presente estudo, de $52 \%$ de melhora da fala, dos quais somente $26 \%$ passaram a apresentar fechamento velofaríngeo adequado após o retalho faríngeo foram relatadas em um estudo com 31 pacientes $^{(18)}$. Resultados similares, com proporção de $49 \%$ de fechamento adequado após o retalho faríngeo e $45 \%$ após a esfincteroplastia foram relatados em um estudo multicêntri$\mathrm{co}^{(19)}$. Proporções superiores, de $93 \%$ de fechamento adequado $^{(20)}, 72 \%$ de normalização da nasalância ${ }^{(21)}, 75 \%$ e $60 \%$ de redução da hipernasalidade e da nasalância ${ }^{(22)}$, foram relatadas 
após o retalho faríngeo e, $79 \%$ de redução da hipernasalidade e $75 \%$ de diminuição do orifício velofaríngeo, verificados à nasoendoscopia, foram relatadas após a esfincteroplastia( ${ }^{(23)}$.

Em outro estudo realizado no Laboratório de Fisiologia ${ }^{(24)}$, em que a técnica fluxo-pressão foi utilizada, juntamente com a avaliação perceptiva e nasométrica, para avaliar os resultados da cirurgia de retalho faríngeo em mais de 200 pacientes com IVF, verificou-se redução da hipernasalidade em $75 \%$ dos pacientes, proporção superior à encontrada no presente estudo. A autora constatou que as avaliações instrumentais confirmaram os resultados da avaliação perceptiva da fala.

Ainda que a comparação entre os diversos estudos tenha sido realizada, fatores como diferentes metodologias empregadas, tamanho da amostra, variação das características consideradas para a seleção da cirurgia, aspectos da fala considerados para análise e variação nos critérios utilizados para definir o sucesso cirúrgico dificultam este tipo de análise. Este problema se agrava, ainda mais, quando os resultados são comparados entre diferentes técnicas cirúrgicas.

Apesar de vários autores terem descrito os efeitos desta cirurgia sobre a fala de indivíduos com IVF, até o momento, nenhum deles analisou as repercussões aerodinâmicas da veloplastia intravelar sobre o mecanismo velofaríngeo, por meio da técnica fluxo-pressão. A maioria dos estudos publicados, sobre os resultados da cirurgia, baseou-se em informações derivadas da avaliação subjetiva da fala e de métodos diretos de avaliação tais como nasofaringoscopia e videofluoroscopia, como já mencionados. Embora a nasofaringoscopia permita a visualização direta das estruturas do esfíncter velofaríngeo durante a fala, apresenta algumas limitações, como o fato de ser um método invasivo e ter a desvantagem de ser subjetivo por não oferecer dados quantitativos, inferindo arbitrariamente no tamanho do gap velofaríngeo.

A despeito da indiscutível importância da avaliação perceptiva no diagnóstico da IVF, especialmente, por ser este um método capaz de identificar os sintomas da IVF e a sua significância clínica, no presente estudo, o fechamento velofaríngeo foi avaliado somente por meio de metodologia objetiva. A técnica fluxo-pressão foi escolhida para este fim por permitir, por si só, avaliar as repercussões aerodinâmicas do fechamento velofaríngeo por meio da medida da área do orifício velofaríngeo e inferir o potencial de fechamento velofaríngeo durante a fala. Este é o primeiro estudo a utilizar esta abordagem para aferir os resultados da veloplastia intravelar no HRAC-USP.

Diferentemente dos métodos diretos, a técnica fluxopressão é um método não invasivo que permite estimar a função velofaríngea por meio da medida simultânea de fluxos e pressões durante a fala. Estudos têm demonstrado a eficácia deste método na avaliação do funcionamento do mecanismo velofaríngeo $^{(18,20,27-29)}$.

Embora a veloplastia intravelar seja considerada um procedimento cirúrgico efetivo para a correção da IVF, em 34\% dos pacientes não se constatou modificação positiva após a cirurgia, os quais permaneceram na condição de fechamento velofaríngeo inadequado ou marginal. Esta proporção é semelhante à de $30 \%$ de IVF permanente relatada por outros autores após a veloplastia intravelar ${ }^{(6)}$, porém, é maior que a de $14 \%$ de insucesso obtida por um outro grupo que avaliou os resultados da veloplastia intravelar modificada pelos próprios autores ${ }^{(10)} \mathrm{e}$ de $25 \%$ após a cirurgia do retalho faríngeo verificada em estudo realizado no Laboratório de Fisiologia ${ }^{(24)}$. Pode-se especular que fatores como falha no diagnóstico pré-operatório ou na escolha do procedimento cirúrgico e problemas de cicatrização tecidual podem estar envolvidos neste resultado, visto que, qualquer técnica cirúrgica para correção da IVF está associada a um risco potencial de insucesso no que se refere à resolução dos problemas de fala ${ }^{(30)}$.

Em resumo, a cirurgia da veloplastia intravelar foi eficiente em melhorar o fechamento velofaríngeo e corrigir os sintomas de fala da IVF. Este foi o primeiro estudo que utilizou abordagem aerodinâmica para aferir os resultados da palatoplastia secundária com veloplastia intravelar. Estudos posteriores deverão ser conduzidos, a fim de correlacionar os achados aerodinâmicos e perceptivos e ainda, aos obtidos com outras técnicas instrumentais como a nasofaringoscopia, a videofluoroscopia e a nasometria. Pretende-se, ainda, verificar a influência de outras variáveis tais como idade na cirurgia, fonoterapia e condição pré-operatória sobre os resultados.

\section{CONCLUSÕES}

Com base nos resultados obtidos sobre o efeito da veloplastia intravelar no fechamento velofaríngeo dos pacientes, avaliado por meio da técnica fluxo-pressão, concluiu-se que a cirurgia levou à melhora do fechamento velofaríngeo na maioria dos pacientes analisados. 


\begin{abstract}
Purpose: To evaluate the effect of intravelar veloplasty on velopharyngeal closure of patients with repaired cleft palate and velopharyngeal insufficiency (VPI), using the pressure-flow technique. Methods: Forty-seven individuals of both genders, with ages between six and 48 years, with repaired cleft palate \pm lip and residual VPI, submitted to intravelar veloplasty. The pressure-flow technique was used to measure the velopharyngeal orifice area during the production of the phoneme /p/ in the word "rampa", before and, in average, 12 months after surgery. Velopharyngeal closure was classified as adequate ( 0 to $\left.0.049 \mathrm{~cm}^{2}\right)$, borderline $(0.050$ to 0.199 $\mathrm{cm}^{2}$ ) or inadequate $\left(\geq 0.200 \mathrm{~cm}^{2}\right)$. Results: After surgery, there was considerable improvement in the velopharyngeal closure degree in $66 \%$ of the cases. From the patients analyzed, $47 \%$ presented adequate closure, $23 \%$, borderline closure and $30 \%$ remained with inadequate closure. Conclusion: These results showed that intravelar veloplasty led to improvement in velopharyngeal closure in most of the patients studied, consequently decreasing the speech symptoms of VPI.
\end{abstract}

Keywords: Cleft palate/surgery; Velopharyngeal insufficiency/surgery; Rhinomanometry/methods; Speech; Palate, soft

\title{
REFERÊNCIAS
}

1. Kummer AW. Velopharyngeal dysfunction (VPD) and resonance disorders. In: Kummer AW. Cleft palate \& craniofacial anomalies: effects of speech and resonance. San Diego: Singular; 2001 p.145-76.

2. Trindade IEK, Genaro KF, Yamashita RP, Miguel HC, Fukushiro AP. Proposta de classificação da função velofaríngea na avaliação perceptivo-auditiva da fala. Pró-Fono. 2005;17(2):259-62.

3. Huang MH, Lee ST, Rajendran K. Anatomic basis of cleft palate and velopharyngeal surgery: implications from a fresh cadaveric study. Plast Reconstr Surg. 1998;101(3):613-27; discussion 628-9.

4. Chait L, Gavron G, Graham C, Noik E, De Aguiar G. Modifying the two-stage cleft palate surgical correction. Cleft Palate Craniofac J. 2002;39(2):226-32.

5. Chen PK, Wu JT, Chen YR, Noordhoff MS. Correction of secundary velopharyngeal insufficiency in cleft palate patients with the Furlow palatoplasty. Plast Reconstr Surg. 1994;94(7):933-41; discussion 942-3.

6. Sie KC, Tampakopoulou DA, Sorom J, Gruss JS Eblen LE. Results with Furlow palatoplasty in management of velopharyngeal insufficiency. Plast Reconstr Surg. 2001;108(1):17-25; discussion 26-9.

7. Johns DF, Rohrich RJ, Awada M. Velopharyngeal incompetence: a guide for clinical evaluation. Plast Reconstr Surg. 2003;112(7):1890-7; quiz 1898,1982 .

8. Hudson DA, Grobbelaar AO, Fernandes DB, Lentin R. Treatment of velopharyngeal incompetence by the Furlow Z-plasty. Ann Plast Surg. 1995;34(1):23-6.

9. Sommerlad BC, Mehendale FV, Birch MJ, Sell D, Hattee C, Harland K. Palate re-repair revisited. Cleft Palate Craniofac J. 2002;39(3):295-307. Comment in: Cleft Palate Craniofac J. 2003;40(1):107; author reply 107-8.

10. Nakamura N, Ogata Y, Sasaguri M, Suzuki A, Kikuta R, Ohishi M. Aerodynamic and cephalometric analyses of velopharyngeal structure and function following re-pushback surgery for secondary correction in cleft palate. Cleft Palate Craniofac J. 2003;40(1):46-53.

11. LaRossa D. The state of the art in cleft palate surgery. Cleft Palate Craniofac J. 2000;37(3):225-8.

12. Deren O, Ayhan M, Tuncel A, Görgü M, Altuntas A, Kutlay R, Erdo an B. The correction of velopharyngeal insufficiency by Furlow palatoplasty in patients older than 3 years undergoing Veau-WardillKilner palatoplasty: a prospective clinical study. Plast Reconstr Surg. 2005;116(1):85-93; discussion 94-6.

13. Noorchashm N, Dudas JR, Ford M, Gastman B, Deleyiannis FW, Vecchione L, et al. Conversion Furlow palatoplasty: salvage of speech after straight-line palatoplasty and "incomplete intravelar veloplasty". Ann Plast Surg. 2006;56(5):505-10.

14. Hassan ME, Askar S. Does palatal muscle reconstruction affect the functional outcome of cleft palate surgery? Plast Reconstr Surg 2007;119(6):1859-65.
15. Andrades P, Espinosa-de-los-Monteros A, Shell DH 4th, Thurston TE, Fowler JS, Xavier ST, et al. The importance of radical intravelar veloplasty during two-flap palatoplasty. Plast Reconstr Surg. 2008;122(4):1121-30.

16. Carvalho ELL. Resultado de fala em pacientes submetidos à palatoplastia secundária associada à veloplastia intravelar [tese]. Bauru: Hospital de Reabilitação de Anomalias Craniofaciais da Universidade de São Paulo; 2006.

17. American Cleft Palate-Craniofacial Association - ACPA. [Internet]. Chapel Hill: American Cleft Palate-Craniofacial Association; 2004. Parameters for evaluation and treatment of patients with cleft lip/ palate or other craniofacial anomalies [Internet]. [cited 2007 Nov 25]. Available from: http://www.acpa-cpf.org/teamcare/Parameters04rev. pdf

18. Smith BE, Skef Z, Cohen M, Dorf DS. Aerodynamic assessment of the results of pharyngeal flap surgery: a preliminary investigation. Plast Reconstr Surg. 1985;76(3):402-10.

19. Abyholm F, D'Antonio L, Davidson Ward SL, Kjøll L, Saeed M, Shaw W, Sloan G, Whitby D, Worhington H, Wyatt R; VPI Surgical Group. Pharyngeal flap and sphincterplasty for velopharyngeal insufficiency have equal outcome at 1 year postoperatively: results of a randomized trial. Cleft Palate Craniofac J. 2005;42(5):501-11.

20. Jarvis BL, Trier WC. The effect of intravelar veloplasty on velopharyngeal competence following pharyngeal flap surgery. Cleft Palate J. 1988;25(4):389-94.

21. Haapanen ML. Nasalance scores in patients with a modified Honig velopharyngeal flap before and after operation. Scand J Plast Reconstr Hand Surg. 1992;26(3):301-5.

22. Zuiani TBB, Trindade IEK, Yamashita RP, Trindade Junior AS. The pharyngeal flap surgery in patients with velopharyngeal insufficiency: perceptual and nasometric speech assessment. Braz J Dysmorphol Speech Disord. 1998;2(1):31-42.

23. Witt PD, D'Antonio LL, Zimmerman GJ, Marsh JL. Sphincter pharyngoplasty: a preoperative and postoperative analysis of perceptual speech characteristics and endoscopic studies of velopharyngeal function. Plast Reconstr Surg. 1994;93(6):1154-68. Comment in: Plast Reconstr Surg. 1995;96(7):1745-6.

24. Fukushiro AP. Análise perceptiva, nasométrica e aerodinâmica da fala de indivíduos submetidos à cirurgia de retalho faríngeo para a correção da insuficiência velofaríngea [tese]. Bauru: Hospital de Reabilitação de Anomalias Craniofaciais da Universidade de São Paulo Hospital de Reabilitação de Anomalias Craniofaciais; 2007.

25. Warren DW, Dubois AB. A pressure-flow technique for measuring velopharyngeal orifice area during continuous speech. Cleft Palate J. 1964;16:52-71.

26. Warren DW. Aerodynamics assessment and procedures to determine extent of velopharyngeal inadequacy. In: Bzoch KR, editor. 
Communicative disorders related to cleft lip and palate. 4th ed. Austin: Pro-Ed; 1997. p. 411-37.

27. Trindade IE, Yamashita RP, Suguimoto RM, Mazzottini R, Trindade AS Jr. Effects of orthognathic surgery on speech and breathing of subjects with cleft lip and palate: acoustic and aerodynamic assessment. Cleft Palate Craniofac J. 2003;40(1):54-64.

28. Markkanen-Leppänen M, Isotalo E, Mäkitie AA, Suominen E, AskoSeliavaara S, Haapanen ML. Speech aerodynamics and nasalance in oral cancer patients treated with microvascular transfers. J Craniofac Surg. 2005;16(6):990-5; discussion 996.

29. Miguel HC, Genaro KF, Trindade IEK. Avaliação perceptiva e instrumental da função velofaríngea na fissura de palato submucosa assintomática. Pro-Fono. 2007;19(1):105-12.

30. Wells MD, Vu TA, Luce EA. Incidence and sequelae of nocturnal respiratory obstruction following posterior pharyngeal flap operation. Ann Plast Surg. 1999;43(3):252-7. 